\title{
Interval-Valued Fuzzy KUS-Ideals in KUS-Algebras
}

\author{
Samy M. Mostafa ${ }^{1}$, Mokhtar A.Abdel Naby ${ }^{2}$, Fayza Abdel Halim², \\ Areej T. Hameed ${ }^{4}$ \\ 1 and 2 Department of Mathematics, Faculty of Education, Ain Shams University, Roxy, Cairo, Egypt \\ 3 and 4 Department of Pure Mathematics, Faculty of Sciences, Ain Shams University, Cairo, Egypt. \\ ${ }^{4}$ Department of Mathematics, College of Education for Girls, University of Kufa, Najaf, Iraq.
}

Abstract: In this paper the notion of interval-valued fuzzy KUS-ideals (briefly i-v fuzzy KUS-ideal) in KUSalgebras is introduced. Several theorems are stated and proved. The image and inverse image of $i$-v fuzzy KUSideals are defined and how the homomorphic images and inverse images of $i-v$ fuzzy KUS-ideals become $i-v$ fuzzy KUS-ideals in KUS-algebras is studied as well.

Keywords: KUS-algebras, fuzzy KUS-ideals, interval-valued fuzzy KUS-sub-algebras, interval-valued fuzzy KUS-ideals in KUS-algebras.

2000 Mathematics Subject Classification: 06F35, 03G25, 03B52, 94D05.

\section{Introduction}

W. A. Dudek and X. Zhang ([2],[3]) studied ideals and congruences of BCC-algebras. C. Prabpayak and U. Leerawat ([5],[6]) introduced a new algebraic structure which is called KU-algebras and investigated some related properties. The concept of a fuzzy set, was introduced by L.A. Zadeh [8]. O.G. Xi [7] applied the concept of fuzzy set to BCK-algebras and gave some of its properties. In [9], L.A. Zadeh made an extension of the concept of fuzzy set by an interval-valued fuzzy set (i.e., a fuzzy set with an interval-valued membership function). This interval-valued fuzzy set is referred to as an i-v fuzzy set. He constructed a method of approximate inference using his i-v fuzzy sets. In [1], R. Biswas defined interval-valued fuzzy subgroups and investigated some elementary properties. Recently S.M. Mostafa, and et al ([4]) introduced a new algebraic structure, called KUS-algebra, They have studied a few properties of these algebras, the notion of KUS-ideals on KUS-algebras was formulated and some of its properties are investigated. In this paper, using the notion of interval-valued fuzzy set by L.A. Zadeh, we introduce the concept of an interval-valued fuzzy KUS-ideals (briefly, i-v fuzzy KUS-ideals) of a KUS-algebra, and study some of their properties. Using an i-v level set of an i-v fuzzy set, we state a characterization of an i-v fuzzy KUS-ideals. We prove that every KUS-ideals of a KUSalgebra X can be realized as an i-v level KUS-ideals of an i-v fuzzy KUS ideals of X. In connection with the notion of homomorphism, we study how the images and inverse images of i-v fuzzy KUS-ideals become i-v fuzzy KUS-ideals.

\section{The Structure of KUS-algebras:}

In this section we include some elementary aspects that are necessary for this paper

Definition 2.1([4]). Let $(\mathrm{X} ; *, 0)$ be an algebra with a single binary operation $(*)$. $\mathrm{X}$ is called a KUS-algebra if it satisfies the following identities:

$\left(\mathrm{kus}_{1}\right):(\mathrm{z} * \mathrm{y}) *(\mathrm{z} * \mathrm{x})=\mathrm{y} * \mathrm{x}$

$\left(\mathrm{kus}_{2}\right): 0 * \mathrm{x}=\mathrm{x}$

$\left(\mathrm{kus}_{3}\right): \mathrm{x} * \mathrm{x}=0$

$\left(\right.$ kus $\left._{4}\right): \mathrm{x} *(\mathrm{y} * \mathrm{z})=\mathrm{y} *(\mathrm{x} * \mathrm{z})$, for any $\mathrm{x}, \mathrm{y}, \mathrm{z} \in \mathrm{X}$

In what follows, let $(\mathrm{X} ; *, 0)$ be denote a KUS-algebra unless otherwise specified.

For brevity we also call $\mathrm{X}$ a KUS-algebra. In $\mathrm{X}$ we can define a binary relation $(\leq)$ by: $\mathrm{x} \leq \mathrm{y}$ if and only if $\mathrm{y}$ $* \mathrm{x}=0$.

Lemma 2.2 ([4]). In any KUS-algebra $(\mathrm{X} ; *, 0)$, the following properties hold: for all $\mathrm{x}, \mathrm{y}, \mathrm{z} \in \mathrm{X}$;

a) $\mathrm{x} * \mathrm{y}=0$ and $\mathrm{y} * \mathrm{x}=0$ imply $\mathrm{x}=\mathrm{y}$,

b) $\mathrm{y} *[(\mathrm{y} * \mathrm{z}) * \mathrm{z}]=0$

c) $(0 * \mathrm{x}) *(\mathrm{y} * \mathrm{x})=\mathrm{y} * 0$

d) $\mathrm{x} \leq \mathrm{y}$ implies that $\mathrm{y} * \mathrm{z} \leq \mathrm{x} * \mathrm{z}$ and $\mathrm{z} * \mathrm{x} \leq \mathrm{z} * \mathrm{y}$,

e) $\mathrm{x} \leq \mathrm{y}$ and $\mathrm{y} \leq \mathrm{z}$ imply $\mathrm{x} \leq \mathrm{z}$,

f) $\mathrm{x} * \mathrm{y} \leq \mathrm{z}$ implies that $\mathrm{z} * \mathrm{y} \leq \mathrm{x}$.

Definition 2.3 ([4]). A nonempty subset I of a KUS-algebra $X$ is called a KUS-ideal of $X$ if it satisfies: for all $x$ $, \mathrm{y}, \mathrm{z} \in \mathrm{X}$,

$\left(\mathrm{Ikus}_{1}\right) \quad(0 \in \mathrm{I})$ 
$\left(\operatorname{Ikus}_{2}\right) \quad(\mathrm{z} * \mathrm{y}) \in \mathrm{I}$ and $(\mathrm{y} * \mathrm{x}) \in \mathrm{I}$ imply $(\mathrm{z} * \mathrm{x}) \in \mathrm{I}$.

Definition 2.4([8]). Let $X$ be a nonempty set, a fuzzy subset $\mu$ in $X$ is a function $\mu: X \rightarrow[0,1]$.

Definition 2.5([4]). Let $X$ be a KUS-algebra and, a fuzzy subset $\mu$ in $X$ is called a fuzzy KUS-sub-algebra of $X$ if $\mu(x * y) \geq \min \{\mu(x), \mu(y)\}$, for all $x, y \in X$.

Definition 2.6([4]). Let $X$ be a KUS-algebra , a fuzzy subset $\mu$ in $X$ is called a fuzzy KUS-ideal of $X$ if it satisfies the following conditions: for all $\mathrm{x}, \mathrm{y}, \mathrm{z} \in \mathrm{X}$,

$\left(\right.$ Fkus $\left._{1}\right) \mu(0) \geq \mu(\mathrm{x})$,

$\left(\right.$ Fkus $\left._{2}\right) \quad \mu(\mathrm{z} * \mathrm{x}) \geq \min \{\mu(\mathrm{z} * \mathrm{y}), \mu(\mathrm{y} * \mathrm{x})\}$

Proposition 2.7([4]). The intersection of any finite sets of fuzzy KUS-ideals of KUS-algebra X is also a fuzzy KUS-ideal .

Definition 2.8([9]). Let $X$ be a set and $\mu$ be a fuzzy subset of $X$, for $t \in[0,1]$, the set $\mu_{t}=\{x \in X \mid \mu(x) \geq$ t\} is called a level subset of $\mu$.

Theorem 2.9([4]). A fuzzy subset $\mu$ of KUS-algebra $X$ is a fuzzy KUS-ideal of $X$ if and only if , for every $t \in$ $[0,1], \mu_{t}$ is either empty or a KUS-ideal of $X$.

Definition 2.10([6]) .Let $(\mathrm{X} ; *, 0)$ and $\left(\mathrm{Y} ; *{ }^{`}, 0^{`}\right)$ be nonempty sets . The mapping $f:(\mathrm{X} ; *, 0) \rightarrow\left(\mathrm{Y} ; *{ }^{\prime}, 0^{\prime}\right)$ is called a homomorphism if it satisfies

$f(\mathrm{x} * \mathrm{y})=f(\mathrm{x}) *^{`} f(\mathrm{y})$ for all $\mathrm{x}, \mathrm{y} \in \mathrm{X}$. The set $\left\{\mathrm{x} \in \mathrm{X} \mid f(\mathrm{x})=0{ }^{\prime}\right\}$ is called the Kernel of $f$ and is denoted by $\operatorname{Ker} f$.

Definition $2.11([6])$. Let $f:(\mathrm{X} ; *, 0) \rightarrow\left(\mathrm{Y} ; *^{*}, 0^{\prime}\right)$ be a mapping from the set $\mathrm{X}$ to a set $\mathrm{Y}$. If $\mu$ is a fuzzy subset of $\mathrm{X}$, then the fuzzy subset $\beta$ of $\mathrm{Y}$ defined by:

$$
f(\mu)(y)=\left\{\begin{array}{l}
\sup \left\{\mu(x): x \in f^{-1}(y)\right\} \quad \text { if } f^{-1}(y)=\{x \in X, f(x)=y\} \neq \phi \\
0 \quad \text { otherwise }
\end{array}\right.
$$

is said to be the image of $\mu$ under $f$.

Similarly if $\beta$ is a fuzzy subset of $\mathrm{Y}$, then the fuzzy subset $\mu=(\beta \circ f)$ in $\mathrm{X}$ (i.e the fuzzy subset defined by $\mu(\mathrm{x})=\beta(f(\mathrm{x}))$ for all $\mathrm{x} \in \mathrm{X})$ is called the pre-image of $\beta$ under $f$.

Theorem 2.12([4]). An into homomorphic pre-image of a fuzzy KUS-ideal is a fuzzy KUS-ideal .

Theorem 2.13([4]). An into homomorphic image of a fuzzy KUS-ideal is a fuzzy KUS-ideal .

\section{Interval-valued fuzzy KUS-ideal of KUS-algebra}

Remark 3.1([9]). An interval-valued fuzzy subset (briefly $\mathrm{i}-\mathrm{v}$ fuzzy subset ) A defined in the set $\mathrm{X}$ is given by $\mathrm{A}=\left\{\left(\mathrm{x},\left[\mu_{\mathrm{A}}^{\mathrm{L}}(\mathrm{x}), \mu_{\mathrm{A}}^{\mathrm{U}}(\mathrm{x})\right]\right)\right\}$, for all $\mathrm{x} \in \mathrm{X}$. (briefly, it is denoted by $\mathrm{A}=\left[\mu_{\mathrm{A}}^{\mathrm{L}}, \mu_{\mathrm{A}}^{\mathrm{U}}\right]$ where $\mu_{\mathrm{A}}^{\mathrm{L}}$ and $\mu_{\mathrm{A}}^{\mathrm{U}}$ are any two fuzzy subsets in $\mathrm{X}$ such that $\mu_{\mathrm{A}}^{\mathrm{L}}(\mathrm{x}) \leq \mu_{\mathrm{A}}^{\mathrm{U}}(\mathrm{x})$ for all $\mathrm{x} \in \mathrm{X}$.

Let $\tilde{\mu}_{\mathrm{A}}(\mathrm{x})=\left[\mu_{\mathrm{A}}^{\mathrm{L}}(\mathrm{x}), \mu_{\mathrm{A}}^{\mathrm{U}}(\mathrm{x})\right]$, for all $\mathrm{x} \in \mathrm{X}$ and let $\mathrm{D}[0,1]$ be denotes the family of all closed sub-interval of $[0,1]$. It is clear that if $\mu_{\mathrm{A}}^{\mathrm{L}}(\mathrm{x})=\mu_{\mathrm{A}}^{\mathrm{U}}(\mathrm{x})=\mathrm{c}$, where

$0 \leq \mathrm{c} \leq 1$, then $\tilde{\mu}_{\mathrm{A}}(\mathrm{x})=[\mathrm{c}, \mathrm{c}]$ in $\mathrm{D}[0,1]$, then $\tilde{\mu}_{\mathrm{A}}(\mathrm{x}) \in[0,1]$, for all $\mathrm{x} \in \mathrm{X}$. Therefore the $\mathrm{i}-\mathrm{v}$ fuzzy subset A is given by :

$$
\mathrm{A}=\left\{\left(\mathrm{x}, \tilde{\mu}_{\mathrm{A}}(\mathrm{x})\right)\right\} \text {, for all } \mathrm{x} \in \mathrm{X} \text { where } \tilde{\mu}_{\mathrm{A}}: \mathrm{X} \rightarrow \mathrm{D}[0,1] .
$$

Now we define the refined minimum (briefly $r$ min) and order " $\leq$ " on elements

$\mathrm{D}_{1}=\left[\mathrm{a}_{1}, \mathrm{~b}_{1}\right]$ and $\mathrm{D}_{2}=\left[\mathrm{a}_{2}, \mathrm{~b}_{2}\right]$ of $\mathrm{D}[0,1]$ as follows:

$r \min \left(D_{1}, D_{2}\right)=\left[\min \left\{a_{1}, a_{2}\right\}, \min \left\{b_{1}, b_{2}\right\}\right], D_{1} \leq D_{2} \Leftrightarrow a_{1} \leq a_{2}$ and $b_{1} \leq b_{2}$. Similarly we can define $(\geq)$ and $(=)$.

In what follows, let X denote a KUS-algebra unless otherwise specified, we begin with the following definition.

Definition 3.2. An i-v fuzzy subset $A$ in $X$ is called an $i-v$ fuzzy KUS-sub-algebra of $X$ if $\tilde{\mu}_{A}(x * y) \geq r$ $\min \left\{\tilde{\mu}_{\mathrm{A}}(\mathrm{x}), \tilde{\mu}_{\mathrm{A}}(\mathrm{y})\right\}$, for all $\mathrm{x}, \mathrm{y} \in \mathrm{X}$.

Example 3.3. Let $X=\{0,1,2,3\}$ in which the operation (as in example (*) be define by the following table: 


\begin{tabular}{|c|c|c|c|c|}
\hline$*$ & $\mathbf{0}$ & $\mathbf{1}$ & $\mathbf{2}$ & $\mathbf{3}$ \\
\hline $\mathbf{0}$ & $\mathbf{0}$ & $\mathbf{1}$ & $\mathbf{2}$ & $\mathbf{3}$ \\
\hline $\mathbf{1}$ & $\mathbf{1}$ & $\mathbf{0}$ & $\mathbf{3}$ & $\mathbf{2}$ \\
\hline $\mathbf{2}$ & $\mathbf{2}$ & $\mathbf{3}$ & $\mathbf{0}$ & $\mathbf{1}$ \\
\hline $\mathbf{3}$ & $\mathbf{3}$ & $\mathbf{2}$ & $\mathbf{1}$ & $\mathbf{0}$ \\
\hline
\end{tabular}

Then $(\mathrm{X} ; *, 0)$ is a KUS-algebra. Define a fuzzy subset $\mu: \mathrm{X} \rightarrow[0,1]$ by

$\mu(x)=\left\{\begin{array}{cc}0.7 & \text { if } x=\{0,1\} \\ 0.3 & \text { otherwise }\end{array} \quad . I_{1}=\{0,1\}\right.$ is a KUS-ideal of X. Routine calculation given that $\mu$ is a fuzzy

KUS-ideal of X. Define $\tilde{\mu}_{\mathrm{A}}(\mathrm{x})$ as follows:

$\tilde{\mu}_{\mathrm{A}}(\mathrm{x})=\left\{\begin{array}{ll}{[0.3,0.9]} & \text { if } x=\{0,1\} \\ {[0.1,0.6]} & \text { otherwise }\end{array}\right.$. It is easy to check that A is an i-v fuzzy

KUS-sub-algebra.

Proposition 3.4. If $A$ is an $i-v$ fuzzy KUS-sub-algebra of $X$, then $\tilde{\mu}_{A}(0) \geq \tilde{\mu}_{A}(x)$, for all $x \in X$.

Proof. For all $\mathrm{x} \in \mathrm{X}$, we have $\tilde{\mu}_{\mathrm{A}}(0)=\tilde{\mu}_{\mathrm{A}}(\mathrm{x} * \mathrm{x}) \geq \mathrm{r} \min \left\{\tilde{\mu}_{\mathrm{A}}(\mathrm{x}), \tilde{\mu}_{\mathrm{A}}(\mathrm{x})\right\}$

$=\mathrm{r} \min \left\{\left[\mu_{\mathrm{A}}^{\mathrm{L}}(\mathrm{x}), \mu_{\mathrm{A}}^{\mathrm{U}}(\mathrm{x})\right],\left[\mu_{\mathrm{A}}^{\mathrm{L}}(\mathrm{x}), \mu_{\mathrm{A}}^{\mathrm{U}}(\mathrm{x})\right]\right\}=\mathrm{r} \min \left\{\left[\mu_{\mathrm{A}}^{\mathrm{L}}(\mathrm{x}), \mu_{\mathrm{A}}^{\mathrm{U}}(\mathrm{x})\right]\right\}=\tilde{\mu}_{\mathrm{A}}(\mathrm{x}) . \Delta$

Proposition 3.5. Let $A$ be an $i-v$ fuzzy KUS-sub-algebra of $X$, if there exist a sequence $\left\{X_{n}\right\}$ in $X$ such that $\lim _{n \rightarrow \infty} \tilde{\mu}_{A}\left(x_{n}\right)=[1,1]$, then $\tilde{\mu}_{A}(0)=[1,1]$.

Proof. By proposition (3.4), we have $\tilde{\mu}_{\mathrm{A}}(0) \geq \tilde{\mu}_{\mathrm{A}}(\mathrm{x})$, for all $\mathrm{x} \in \mathrm{X}$. Then $\tilde{\mu}_{\mathrm{A}}(0) \geq \tilde{\mu}_{\mathrm{A}}\left(\mathrm{x}_{\mathrm{n}}\right)$, for every positive integer $\mathrm{n}$, Consider the inequality $[1,1] \geq \tilde{\mu}_{\mathrm{A}}(0) \geq \lim _{\mathrm{n} \rightarrow \infty} \tilde{\mu}_{\mathrm{A}}\left(\mathrm{x}_{\mathrm{n}}\right)=[1,1]$. Hence $\tilde{\mu}_{\mathrm{A}}(0)=[1,1] . \Delta$

Definition 3.6. An i-v fuzzy subset $A=\left\{\left(x, \tilde{\mu}_{A}(x)\right)\right\}, x \in X$ in KUS-algebra $X$ is called an interval-valued fuzzy KUS-ideal (i-v fuzzy KUS-ideal, in short) if it satisfies the following conditions:

$\left(\mathrm{A}_{1}\right) \quad \tilde{\mu}_{\mathrm{A}}(0) \geq \tilde{\mu}_{\mathrm{A}}(\mathrm{x})$,

$\left(\mathrm{A}_{2}\right) \quad \tilde{\mu}_{\mathrm{A}}(\mathrm{z} * \mathrm{x}) \geq \mathrm{r} \min \left\{\tilde{\mu}_{\mathrm{A}}(\mathrm{z} * \mathrm{y}), \tilde{\mu}_{\mathrm{A}}(\mathrm{y} * \mathrm{x})\right\}$, for all $\mathrm{x}, \mathrm{y}, \mathrm{z} \in \mathrm{X}$.

Example 3.7. Let $X=\{0,1,2,3\}$ as in example (3.3). Define $\tilde{\mu}_{\mathrm{A}}$ (x) as follows:

$\tilde{\mu}_{\mathrm{A}}(\mathrm{x})=\left\{\begin{array}{ll}{[0.3,0.9]} & \text { if } x=\{0,1\} \\ {[0.1,0.6]} & \text { otherwise }\end{array}\right.$. It is easy to check that $\mathrm{A}$ is an $\mathrm{i}-\mathrm{v}$ fuzzy KUS-ideal of $\mathrm{X}$.

Theorem 3.8. An i-v fuzzy subset $A=\left[\mu_{A}^{L}, \mu_{A}^{U}\right]$ in $X$ is an i-v fuzzy KUS-ideal of $X$ if and only if $\mu_{A}^{L}$ and $\mu_{\mathrm{A}}^{\mathrm{U}}$ are fuzzy KUS-ideals of X.

Proof. If $\mu_{\mathrm{A}}^{\mathrm{L}}$ and $\mu_{\mathrm{A}}^{\mathrm{U}}$ are fuzzy KUS-ideals of $\mathrm{X}$. For any $\mathrm{x}, \mathrm{y}, \mathrm{z} \in \mathrm{X}$. Observe $\tilde{\mu}_{\mathrm{A}}\left(\mathrm{z}^{*} \mathrm{x}\right)=$ $\left[\mu_{\mathrm{A}}^{\mathrm{L}}(\mathrm{z} * \mathrm{x}), \mu_{\mathrm{A}}^{\mathrm{U}}(\mathrm{z} * \mathrm{x})\right]$

$$
\begin{aligned}
& \geq\left[\min \left\{\mu_{\mathrm{A}}^{\mathrm{L}}(\mathrm{z} * \mathrm{y}), \mu_{\mathrm{A}}^{\mathrm{L}}(\mathrm{y} * \mathrm{x})\right\}, \min \left\{\mu_{\mathrm{A}}^{\mathrm{U}}(\mathrm{z} * \mathrm{y}), \mu_{\mathrm{A}}^{\mathrm{U}}(\mathrm{y} * \mathrm{x})\right\}\right] \\
& =\mathrm{r} \min \left\{\left[\mu_{\mathrm{A}}^{\mathrm{L}}(\mathrm{z} * \mathrm{y}), \mu_{\mathrm{A}}^{\mathrm{U}}(\mathrm{z} * \mathrm{y})\right],\left[\mu_{\mathrm{A}}^{\mathrm{L}}(\mathrm{y} * \mathrm{x}), \mu_{\mathrm{A}}^{\mathrm{U}}(\mathrm{y} * \mathrm{x})\right]\right\} \\
& =\mathrm{r} \min \left\{\tilde{\mu}_{\mathrm{A}}(\mathrm{z} * \mathrm{y}), \tilde{\mu}_{\mathrm{A}}(\mathrm{y} * \mathrm{x})\right] .
\end{aligned}
$$

From what was mentioned above we can conclude that $\mathrm{A}$ is an $\mathrm{i}-\mathrm{v}$ fuzzy KUS-ideal of $\mathrm{X}$.

Conversely, suppose that $A$ is an i-v fuzzy KUS-ideal of $X$. For all $x, y, z \in X$ we have $\left[\mu_{A}^{L}(z * x)\right.$,

$\left.\mu_{\mathrm{A}}^{\mathrm{U}}(\mathrm{z} * \mathrm{x})\right]=\tilde{\mu}_{\mathrm{A}}(\mathrm{z} * \mathrm{x}) \geq \mathrm{r} \min \left\{\tilde{\mu}_{\mathrm{A}}(\mathrm{z} * \mathrm{y}), \tilde{\mu}_{\mathrm{A}}(\mathrm{y} * \mathrm{x})\right\}$

$=\mathrm{r} \min \left\{\left[\mu_{\mathrm{A}}^{\mathrm{L}}(\mathrm{z} * \mathrm{y}), \mu_{\mathrm{A}}^{\mathrm{U}}(\mathrm{z} * \mathrm{y})\right],\left[\mu_{\mathrm{A}}^{\mathrm{L}}(\mathrm{y} * \mathrm{x}), \mu_{\mathrm{A}}^{\mathrm{U}}(\mathrm{y} * \mathrm{x})\right]\right\}$ 
$=\left[\min \left\{\mu_{\mathrm{A}}^{\mathrm{L}}(\mathrm{z} * \mathrm{y}), \mu_{\mathrm{A}}^{\mathrm{L}}(\mathrm{y} * \mathrm{x})\right\}, \min \left\{\mu_{\mathrm{A}}^{\mathrm{U}}(\mathrm{z} * \mathrm{y}), \mu_{\mathrm{A}}^{\mathrm{U}}(\mathrm{y} * \mathrm{x})\right\}\right]$. Therefore, $\mu_{\mathrm{A}}^{\mathrm{L}}(\mathrm{z} * \mathrm{x}) \geq$ $\min \left\{\mu_{\mathrm{A}}^{\mathrm{L}}(\mathrm{z} * \mathrm{y}), \mu_{\mathrm{A}}^{\mathrm{L}}(\mathrm{y} * \mathrm{x})\right\}$ and

$$
\mu_{\mathrm{A}}^{\mathrm{U}}(\mathrm{z} * \mathrm{x}) \geq \min \left\{\mu_{\mathrm{A}}^{\mathrm{U}}(\mathrm{z} * \mathrm{y}), \mu_{\mathrm{A}}^{\mathrm{U}}(\mathrm{y} * \mathrm{x})\right\} \text {. }
$$

Hence, we get that $\mu_{\mathrm{A}}^{\mathrm{L}}$ and $\mu_{\mathrm{A}}^{\mathrm{U}}$ are fuzzy KUS-ideals of X. $\triangle$

Theorem 3.9. Let $A_{1}$ and $A_{2}$ be $i-v$ fuzzy KUS-ideals of a KUS-algebra $X$. Then $A_{1} \cap A_{2}$ is an i-v fuzzy KUS-ideal of X.

Proof. $\tilde{\mu}_{\mathrm{A}_{1} \cap \mathrm{A}_{2}}(0)=\left[\mu_{\mathrm{A}_{1} \cap \mathrm{A} 2}^{\mathrm{L}}(0), \mu_{\mathrm{A}_{1} \cap A_{2}}^{\mathrm{U}}(0)\right] \geq\left[\mu_{\mathrm{A}_{1} \cap A 2}^{\mathrm{L}}(\mathrm{x}), \mu_{\mathrm{A}_{1} \cap \mathrm{A}_{2}}^{\mathrm{U}}(\mathrm{x})\right]=\tilde{\mu}_{\mathrm{A}_{1} \cap \mathrm{A}_{2}}(\mathrm{x})$.

Suppose $x, y, z \in X$ such that $\left(z^{*} y\right) \in A_{1} \cap A_{2}$ and $\left(y^{*} x\right) \in A_{1} \cap A_{2}$.

Since $A_{1}$ and $A_{2}$ are i-v fuzzy KUS-ideals of $X$, then by the theorem (3.8), we get

$$
\begin{aligned}
& \tilde{\mu}_{\mathrm{A}_{1} \cap \mathrm{A}_{2}}(\mathrm{z} * \mathrm{x})=\left[\mu_{\mathrm{A}_{1} \cap \mathrm{A} 2}^{\mathrm{L}}(\mathrm{z} * \mathrm{x}), \mu_{\mathrm{A}_{1} \cap \mathrm{A}_{2}}^{\mathrm{U}}(\mathrm{z} * \mathrm{x})\right] \\
& \quad=\left[\min \left\{\mu_{\mathrm{A}_{1} \cap \mathrm{A} 2}^{\mathrm{L}}(\mathrm{z} * \mathrm{y}), \mu_{\mathrm{A}_{1} \cap \mathrm{A} 2}^{\mathrm{L}}(\mathrm{y} * \mathrm{x})\right\}, \min \left\{\mu_{\mathrm{A}_{1} \cap \mathrm{A}_{2}}^{\mathrm{U}}(\mathrm{z} * \mathrm{y}), \mu_{\mathrm{A}_{1} \cap \mathrm{A}_{2}}^{\mathrm{U}}(\mathrm{y} * \mathrm{x})\right\}\right] \\
& \quad=\left[\min \left\{\mu_{\mathrm{A}_{1} \cap \mathrm{A} 2}^{\mathrm{L}}(\mathrm{z} * \mathrm{y}), \mu_{\mathrm{A}_{1} \cap \mathrm{A}_{2}}^{\mathrm{U}}(\mathrm{z} * \mathrm{y})\right\}, \min \left\{\mu_{\mathrm{A}_{1} \cap \mathrm{A} 2}^{\mathrm{L}}(\mathrm{y} * \mathrm{x}), \mu_{\mathrm{A}_{1} \cap \mathrm{A}_{2}}^{\mathrm{U}}(\mathrm{y} * \mathrm{x})\right\}\right] \\
& \left.\quad=\operatorname{rmin}\left\{\tilde{\mu}_{\mathrm{A}_{1} \cap \mathrm{A}_{2}}(\mathrm{z} * \mathrm{y}), \tilde{\mu}_{\mathrm{A}_{1} \cap \mathrm{A}_{2}}(\mathrm{y} * \mathrm{x})\right\}\right] .
\end{aligned}
$$

Corollary 3.10. Let $\left\{A_{i} \mid i \in \Lambda\right\}$ be a family of $i-v$ fuzzy KUS-ideal of $X$. Then $\bigcap_{i \in \wedge} A_{i}$ is also an i-v fuzzy KUS-ideal of $X$.

Theorem 3.11. Let $X$ be a KUS-algebra and $A$ be an i-v fuzzy subset in $X$. Then $A$ is an i-v fuzzy KUSideal of $\mathrm{X}$ if and only if the nonempty set

$$
\tilde{U}\left(A ;\left[\delta_{1}, \delta_{2}\right]\right):=\left\{x \in X \mid \tilde{\mu}_{A}(x) \geq\left[\delta_{1}, \delta_{2}\right]\right\} \text { is a KUS-ideal of } \mathrm{X} \text {, for every }
$$

$\left[\delta_{1}, \delta_{2}\right] \in \mathrm{D}[0,1]$. We call $\tilde{\mathrm{U}}\left(\mathrm{A} ;\left[\delta_{1}, \delta_{2}\right]\right)$ the i-v level KUS-ideal of $\mathrm{A}$.

Proof. Assume that $\mathrm{A}$ is an i-v fuzzy KUS-ideal of $\mathrm{X}$ and let $\left[\delta_{1}, \delta_{2}\right] \in \mathrm{D}[0,1]$ be such that $(\mathrm{z} * \mathrm{y}),(\mathrm{y} * \mathrm{x}) \in \tilde{\mathrm{U}}\left(\mathrm{A} ;\left[\delta_{1}, \delta_{2}\right]\right)$, then $\tilde{\mu}_{\mathrm{A}}(\mathrm{z} * \mathrm{x}) \geq \mathrm{r} \min \left\{\tilde{\mu}_{\mathrm{A}}(\mathrm{z} * \mathrm{y}), \tilde{\mu}_{\mathrm{A}}(\mathrm{y} * \mathrm{x})\right\} \geq \mathrm{r} \min \left\{\left[\delta_{1}, \delta_{2}\right],\left[\delta_{1}, \delta_{2}\right]\right\}=\left[\delta_{1}, \delta_{2}\right]$ and so $(\mathrm{z} * \mathrm{x}) \in \tilde{\mathrm{U}}$ (A ; $\left.\left[\delta_{1}, \delta_{2}\right]\right)$. Then $\tilde{U}\left(\mathrm{~A} ;\left[\delta_{1}, \delta_{2}\right]\right)$ the i-v level KUS-ideal of A.

Conversely, assume that $\tilde{\mathrm{U}}\left(\mathrm{A} ;\left[\delta_{1}, \delta_{2}\right]\right) \neq \varnothing$ is a KUS-ideal of $\mathrm{X}$, for every $\left[\delta_{1}, \delta_{2}\right] \in \mathrm{D}[0,1]$.In the contrary, suppose that there exist $\mathrm{x}_{0}, \mathrm{y}_{0}, \mathrm{z}_{0} \in \mathrm{X}$, such that

$$
\tilde{\mu}_{\mathrm{A}}\left(\mathrm{z}_{0} * \mathrm{x}_{0}\right)<\mathrm{r} \min \left\{\tilde{\mu}_{\mathrm{A}}\left(\mathrm{z}_{0} * \mathrm{y}_{0}\right), \tilde{\mu}_{\mathrm{A}}\left(\mathrm{y}_{0} * \mathrm{x}_{0}\right)\right\} .
$$

Let $\tilde{\mu}_{\mathrm{A}}\left(\mathrm{z}_{0} * \mathrm{y}_{0}\right)=\left[\gamma_{1}, \gamma_{2}\right], \tilde{\mu}_{\mathrm{A}}\left(\mathrm{y}_{0} * \mathrm{x}_{0}\right)=\left[\gamma_{3}, \gamma_{4}\right]$ and $\tilde{\mu}_{\mathrm{A}}\left(\mathrm{z}_{0} * \mathrm{x}_{0}\right)=\left[\delta_{1}, \delta_{2}\right]$. If $\left[\delta_{1}, \delta_{2}\right]<\mathrm{r} \min \left\{\left[\gamma_{1}, \gamma_{2}\right],\left[\gamma_{3}, \gamma_{4}\right]\right\}=\min \left\{\min \left\{\gamma_{1}, \gamma_{2}\right\}, \min \left\{\gamma_{3}, \gamma_{4}\right\}\right\}$.

So $\delta_{1}<\min \left\{\gamma_{1}, \gamma_{2}\right\}$ and $\delta_{2}<\min \left\{\gamma_{3}, \gamma_{4}\right\}$. Consider

$$
\left.\left[\lambda_{1}, \lambda_{2}\right]=\frac{1}{2}\left\{\tilde{\mu}_{\mathrm{A}}\left(\mathrm{z}_{0} * \mathrm{x}_{0}\right)+\mathrm{r} \min \left\{\tilde{\mu}_{\mathrm{A}}\left(\mathrm{z}_{0} * \mathrm{y}_{0}\right)\right), \tilde{\mu}_{\mathrm{A}}\left(\mathrm{y}_{0} * \mathrm{x}_{0}\right)\right\}\right\}
$$

We find that

$$
\begin{aligned}
{\left[\lambda_{1}, \lambda_{2}\right]=} & \frac{1}{2}\left\{\left[\delta_{1}, \delta_{2}\right]+\mathrm{r} \min \left\{\left[\gamma_{1}, \gamma_{2}\right],\left[\gamma_{3}, \gamma_{4}\right]\right\}\right\} \\
& =\frac{1}{2}\left\{\left(\delta_{1}+\min \left\{\gamma_{1}, \gamma_{3}\right\}\right),\left(\delta_{2}+\min \left\{\gamma_{2}, \gamma_{4}\right\}\right)\right] .
\end{aligned}
$$

Therefore $\min \left\{\gamma_{1}, \gamma_{3}\right\}>\lambda_{1}=\frac{1}{2}\left(\delta_{1}+\min \left\{\gamma_{1}, \gamma_{3}\right\}\right)>\delta_{1}$, 


$$
\min \left\{\gamma_{2}, \gamma_{4}\right\}>\lambda_{2}=\frac{1}{2}\left(\delta_{2}+\min \left\{\gamma_{2}, \gamma_{4}\right\}\right)>\delta_{2}
$$

Hence $\left[\min \left\{\gamma_{1}, \gamma_{3}\right\}, \min \left\{\gamma_{2}, \gamma_{4}\right\}\right]>\left[\lambda_{1}, \lambda_{2}\right]>\left[\delta_{1}, \delta_{2}\right]=\tilde{\mu}_{\mathrm{A}}\left(\mathrm{z}_{0} * \mathrm{x}_{0}\right)$,

so that, $\left(\mathrm{z}_{0} * \mathrm{x}_{0}\right) \notin \tilde{\mathrm{U}}\left(\mathrm{A} ;\left[\lambda_{1}, \lambda_{2}\right]\right)$. which is a contradiction, since

$$
\tilde{\mu}_{\mathrm{A}}\left(\mathrm{z}_{0} * \mathrm{y}_{0}\right)=\left[\gamma_{1}, \gamma_{2}\right] \geq\left[\min \left\{\gamma_{1}, \gamma_{3}\right\}, \min \left\{\gamma_{2}, \gamma_{4}\right]>\left[\lambda_{1}, \lambda_{2}\right]\right. \text {. }
$$

$\tilde{\mu}_{\mathrm{A}}\left(\mathrm{y}_{0} * \mathrm{x}_{0}\right)=\left[\gamma_{3}, \gamma_{4}\right] \geq\left[\min \left\{\gamma_{1}, \gamma_{3}\right\}, \min \left\{\gamma_{2}, \gamma_{4}\right]>\left[\lambda_{1}, \lambda_{2}\right]\right.$, imply that

$\left(\mathrm{z}_{0} * \mathrm{y}_{0}\right),\left(\mathrm{y}_{0} * \mathrm{x}_{0}\right) \in \tilde{\mathrm{U}}\left(\mathrm{A} ;\left[\lambda_{1}, \lambda_{2}\right]\right)$. Then

$\tilde{\mu}_{\mathrm{A}}(\mathrm{z} * \mathrm{x}) \geq \mathrm{r} \min \left\{\tilde{\mu}_{\mathrm{A}}(\mathrm{z} * \mathrm{y}), \tilde{\mu}_{\mathrm{A}}(\mathrm{y} * \mathrm{x})\right\}$, for all $\mathrm{x}, \mathrm{y}, \mathrm{z} \in \mathrm{X}$.

Theorem 3.12. Every KUS-ideal of a KUS-algebra $X$ can be realized as an i-v level KUS-ideal of an i-v fuzzy KUS-ideal of $X$.

Proof. Let $\mathrm{Y}$ be a KUS-ideal of $\mathrm{X}$ and let $\mathrm{A}$ be an i-v fuzzy subset on $\mathrm{X}$ defined by

$$
\tilde{\mu}_{\mathrm{A}}(\mathrm{x})=\left\{\begin{array}{lr}
{\left[\alpha_{1}, \alpha_{2}\right]} & \text { if } x \in y \\
{[0,0]} & \text { otherwise }
\end{array}\right.
$$

Where $\alpha_{1}, \alpha_{2} \in[0,1]$ with $\alpha_{1}<\alpha_{2}$. It is clear that $\tilde{U}\left(A ;\left[\alpha_{1}, \alpha_{2}\right]\right)=\mathrm{Y}$. We show that A is an i-v fuzzy KUS-ideal of $X$. Let $\mathrm{x}, \mathrm{y}, \mathrm{z} \in \mathrm{X}$.

If $(\mathrm{z} * \mathrm{y}),(\mathrm{y} * \mathrm{x}) \in \mathrm{Y}$, then $(\mathrm{z} * \mathrm{x}) \in \mathrm{Y}$, and therefore

$\left.\tilde{\mu}_{\mathrm{A}}(\mathrm{z} * \mathrm{x})=\left[\alpha_{1}, \alpha_{2}\right]=\mathrm{r} \min \left\{\left[\alpha_{1}, \alpha_{2}\right],\left[\alpha_{1}, \alpha_{2}\right]\right\}=\mathrm{r} \min \left\{\tilde{\mu}_{\mathrm{A}}(\mathrm{z} * \mathrm{y})\right), \tilde{\mu}_{\mathrm{A}}(\mathrm{y} * \mathrm{x})\right\}$.

If $(\mathrm{z} * \mathrm{y}),(\mathrm{y} * \mathrm{x}) \notin \mathrm{Y}$, then $\tilde{\mu}_{\mathrm{A}}(\mathrm{z} * \mathrm{y})=[0,0]=\tilde{\mu}_{\mathrm{A}}(\mathrm{y} * \mathrm{x})$ and so

$$
\tilde{\mu}_{\mathrm{A}}(\mathrm{z} * \mathrm{x}) \geq[0,0]=\mathrm{r} \min \{[0,0],[0,0]\}=\mathrm{r} \min \left\{\tilde{\mu}_{\mathrm{A}}(\mathrm{z} * \mathrm{y}), \tilde{\mu}_{\mathrm{A}}(\mathrm{y} * \mathrm{x})\right\},
$$

If $(\mathrm{z} * \mathrm{y}) \in \mathrm{Y}$ and $(\mathrm{y} * \mathrm{x}) \notin \mathrm{Y}$, then $\tilde{\mu}_{\mathrm{A}}(\mathrm{z} * \mathrm{y})=\left[\alpha_{1}, \alpha_{2}\right]$ and $\tilde{\mu}_{\mathrm{A}}(\mathrm{y} * \mathrm{x})=[0,0]$, then $\tilde{\mu}_{\mathrm{A}}(\mathrm{z} * \mathrm{x}) \geq[0,0]$ $=r \min \left\{\left[\alpha_{1}, \alpha_{2}\right],[0,0]\right\}=r \min \left\{\tilde{\mu}_{\mathrm{A}}(\mathrm{z} * \mathrm{y}), \tilde{\mu}_{\mathrm{A}}(\mathrm{y} * \mathrm{x})\right\}$.

Similarly for the case $(\mathrm{z} * \mathrm{y}) \notin \mathrm{Y}$ and $(\mathrm{y} * \mathrm{x}) \in \mathrm{Y}$ we get

$$
\left.\tilde{\mu}_{\mathrm{A}}(\mathrm{z} * \mathrm{x}) \geq \mathrm{r} \min \left\{\tilde{\mu}_{\mathrm{A}}(\mathrm{z} * \mathrm{y})\right), \tilde{\mu}_{\mathrm{A}}(\mathrm{y} * \mathrm{x})\right\} \text {. }
$$

Therefore $\mathrm{A}$ is an $\mathrm{i}-\mathrm{v}$ fuzzy KUS-ideal of $\mathrm{X}$, the proof is complete. $\triangle$

Proposition 3.13. Let $X$ be a KUS-algebra, B be a fuzzy subset on $X$ and let $A$ be an i-v fuzzy subset on $X$ defined by $\tilde{\mu}_{\mathrm{A}}(\mathrm{x})=\left\{\begin{array}{l}{\left[\alpha_{1}, \alpha_{2}\right] \quad \text { if } \mathrm{x} \in \mathrm{y}} \\ {[0,0] \quad \text { otherwise }}\end{array}\right.$. Where

$\alpha_{1}, \alpha_{2} \in(0,1]$ with $\alpha_{1}<\alpha_{2}$. If $A$ is an i-v fuzzy KUS-sub-algebra of $X$, then $B$ is a fuzzy KUS- subalgebra of X.

Proof. Clear.๑

Theorem 3.14. If $A$ is an $i-v$ fuzzy KUS-ideal of $X$, then the set $X_{\tilde{\mathrm{M}}_{\mathrm{A}}}:=\left\{\mathrm{x} \in \mathrm{X} \mid \tilde{\mu}_{\mathrm{A}}(\mathrm{x})=\tilde{\mu}_{\mathrm{A}}(0)\right\}$ is a KUS-ideal of $\mathrm{X}$.

Proof. Let $(\mathrm{z} * \mathrm{y}),(\mathrm{y} * \mathrm{x}) \in \mathrm{X}_{\tilde{\mathrm{M}}_{\mathrm{A}}}$. Then $\tilde{\mu}_{\mathrm{A}}(\mathrm{z} * \mathrm{y})=\tilde{\mu}_{\mathrm{A}}(0)=\tilde{\mu}_{\mathrm{A}}(\mathrm{y} * \mathrm{x})$, and so $\tilde{\mu}_{\mathrm{A}}(\mathrm{z} * \mathrm{x}) \geq \mathrm{r} \min \left\{\tilde{\mu}_{\mathrm{A}}(\mathrm{z} * \mathrm{y}), \tilde{\mu}_{\mathrm{A}}(\mathrm{y} * \mathrm{x})\right\}=\mathrm{r} \min \left\{\tilde{\mu}_{\mathrm{A}}(0), \tilde{\mu}_{\mathrm{A}}(0)\right\}=\tilde{\mu}_{\mathrm{A}}(0)$.

Combining this with condition (1) of definition (3.6), we get $\tilde{\mu}_{\mathrm{A}}(\mathrm{z} * \mathrm{x})=\tilde{\mu}_{\mathrm{A}}(0)$, that is $(\mathrm{z} * \mathrm{x}) \in \mathrm{X}_{\tilde{\mathrm{M}}_{\mathrm{A}}}$. Hence $\mathrm{X}_{\tilde{\mathrm{M}}_{\mathrm{A}}}$ is a KUS-ideal of X. $\bullet$

\section{Homomorphism of KUS-algebra}

Definition 4.1 ([1]). Let $f:(\mathrm{X} ; *, 0) \rightarrow\left(\mathrm{Y} ; *^{*}, 0^{\prime}\right)$ be a mapping from set $\mathrm{X}$ into a set $\mathrm{Y}$. let $\mathrm{B}$ be an i-v fuzzy subset in $\mathrm{Y}$. Then the inverse image of $\mathrm{B}$, denoted by $\quad f^{-1}(\mathrm{~B})$, is an i-v fuzzy subset in $\mathrm{X}$ with the membership function given by 
$\mu_{\mathrm{f}^{-1}(\mathrm{~B})}(\mathrm{x})=\tilde{\mu}_{\mathrm{B}}(f(\mathrm{x}))$, for all $\mathrm{x} \in \mathrm{X}$.

Proposition 4.2 ([1]). Let $f$ be a mapping from set $\mathrm{X}$ into a set $\mathrm{Y}$, let $\mathrm{m}=\left[\mathrm{m}^{\mathrm{L}}, \mathrm{m}^{\mathrm{u}}\right]$, and $\mathrm{n}=\left[\mathrm{n}^{\mathrm{L}}, \mathrm{n}^{\mathrm{u}}\right]$ be $\mathrm{i}-\mathrm{v}$ fuzzy subsets in $\mathrm{X}$ and $\mathrm{Y}$ respectively. Then

(1) $f^{-1}(\mathrm{n})=\left[f^{-1}\left(\mathrm{n}^{\mathrm{L}}\right), f^{-1}\left(\mathrm{n}^{\mathrm{u}}\right)\right]$,

(2) $f(\mathrm{~m})=\left[f\left(\mathrm{~m}^{\mathrm{L}}\right), f\left(\mathrm{~m}^{\mathrm{u}}\right)\right]$.

Theorem 4.3. Let $f$ be homomorphism from a KUS-algebra X into a KUS-algebra $\mathrm{Y}$. If B is an i-v fuzzy KUS-ideal of $\mathrm{Y}$, then the inverse image $f^{-1}(\mathrm{~B})$ of B is an i-v fuzzy KUS-ideal of X.

Proof. Since $B=\left[\mu_{B}^{L}, \mu_{B}^{u}\right]$ is an i-v fuzzy KUS-ideal of $Y$, it follows that from theorem (3.8), that $\left(\mu_{B}^{L}\right.$ ) and $\left(\mu_{\mathrm{B}}^{\mathrm{u}}\right)$ are fuzzy KUS-ideals of Y. Using theorem (2.12), we know $f^{-1}\left(\mu_{\mathrm{B}}^{\mathrm{L}}\right)$ and $f^{-1}\left(\mu_{\mathrm{B}}^{\mathrm{u}}\right)$ are fuzzy KUS-ideals of X. Hence by proposition (4.2), we conclude that $f^{-1}(\mathrm{~B})=\left[f^{-1}\left(\mu_{\mathrm{B}}^{\mathrm{L}}\right), f^{-1}\left(\mu_{\mathrm{B}}^{\mathrm{u}}\right)\right]$ is an $\mathrm{i}-\mathrm{v}$ fuzzy KUS-ideal of $\mathrm{X}$.

Definition 4.4 ([9]).Let $f$ be a mapping from a set $\mathrm{X}$ into a set $\mathrm{Y}$. let $\mathrm{A}$ be a an i-v fuzzy set in $\mathrm{X}$. then the image of $\mathrm{A}$, denoted by $f(\mathrm{~A})$, is the $\mathrm{i}-\mathrm{v}$ fuzzy subset in $\mathrm{Y}$ with membership function denoted by :

$$
\tilde{\mu}_{\mathrm{f}(\mathrm{A})}(\mathrm{x})=\left\{\begin{array}{ll}
\sup _{\mathrm{z} \in \mathrm{f}^{-1}(\mathrm{y})} \quad \tilde{\mu}_{\mathrm{A}}(\mathrm{z}) \quad \text { if } \mathrm{f}^{-1}(\mathrm{y}) \neq \phi, \mathrm{y} \in \mathrm{Y} \\
{[0,0] \quad \text { otherwise }}
\end{array}\right. \text {, }
$$

where $f^{-1}(\mathrm{y}):=\{\mathrm{x} \in \mathrm{X} \mid f(\mathrm{x})=\mathrm{y}\}$.

Theorem 4.5. Let $f$ be a homomorphism from a KUS-algebra $\mathrm{X}$ into a KUS-algebra $\mathrm{Y}$. If $\mathrm{A}$ is an i-v fuzzy KUS-ideal of X, then $f(\mathrm{~A})$ of $\mathrm{A}$ is an i-v fuzzy KUS-ideal of $\mathrm{Y}$.

Proof. Assume that $A=\left[\mu_{A}^{L}, \mu_{A}^{u}\right]$ is an i-v fuzzy KUS-ideal of X. it follows that from theorem (3.8), that $\left(\mu_{\mathrm{A}}^{\mathrm{L}}\right)$ and $\left(\mu_{\mathrm{A}}^{\mathrm{u}}\right)$ are fuzzy KUS-ideals of X. Using theorem (2.13), that the images $f\left(\mu_{\mathrm{A}}^{\mathrm{L}}\right)$ and $f$ $\left(\mu_{\mathrm{A}}^{\mathrm{u}}\right)$ are fuzzy KUS-ideal of Y. Hence by proposition (4.2), we conclude that $f(\mathrm{~A})=\left[f\left(\mu_{\mathrm{A}}^{\mathrm{L}}\right), f\left(\mu_{\mathrm{A}}^{\mathrm{u}}\right)\right]$ is an i-v fuzzy KUS-ideal of $\mathrm{Y} . \bullet$

\section{References}

[1] Biswas R., Rosenfeld's fuzzy subgroups with interval valued membership, function, Fuzzy Sets and ystems , vol.63, no.1 (1994),8790

[2] Dudek W. A. and Zhang X., On ideal and congruences in BCC-algebras, Czechoslovak Math. Journal, vol.48, no. 123 (1998), $21-29$.

[3] Dudek W. A., On proper BCC-algebras, Bull. Ins. Math. Academic Science, vol. 20 (1992),137-150.

[4] Mostafa S. M., Abdel Naby M. A. , Abdel-Halim F. and Hameed A. T., Fuzzy KUS-ideals in KUS-algebras. To appear .

[5] Prabpayak C. and Leerawat U. , On ideals and congurences in KU-algebras , scientia magna journal , vol.5, no .1 (2009) , 54-57 .

[6] Prabpayak C. and Leerawat U., On isomorphisms of KU-algebras , scientia magna journal, vol.5, no .3 (2009), 25-31 .

[7] Xi O. G., Fuzzy BCK-algebra, Math. Japon. , vol.36 (1991) 935-942.

[8] Zadeh L. A., Fuzzy sets, Inform. And Control, vol. 8 (1965) 338-353.

[9] Zadeh L. A., The concept of a linguistic variable and its application to approximate I, Information Sci. And Control , vol.8 (1975) , 199-249. 